\title{
Segmental Testicular Infarction - Is Conservative Management Feasible?
}

\section{Introduction}

Segmental testicular infarction is a relatively uncommon and benign finding. It occurs predominantly in the second and third decade of life. The main symptoms are unilateral and scrotal as seen in more common conditions such as testicular torsion, epididymitis, orchitis, or blunt trauma.

The primary testis investigation method is ultrasonography (US) with color Doppler. Normally segmental testicular infarction is seen as a round or wedge-shaped hypoecho- ic lesion within the testicle in combination with absent or low blood flow. In contrast, vascular testicular lesions are often malignant. However, US alone cannot rule out testicular neoplasms, since hypovascularity of testicular tumors has been described.
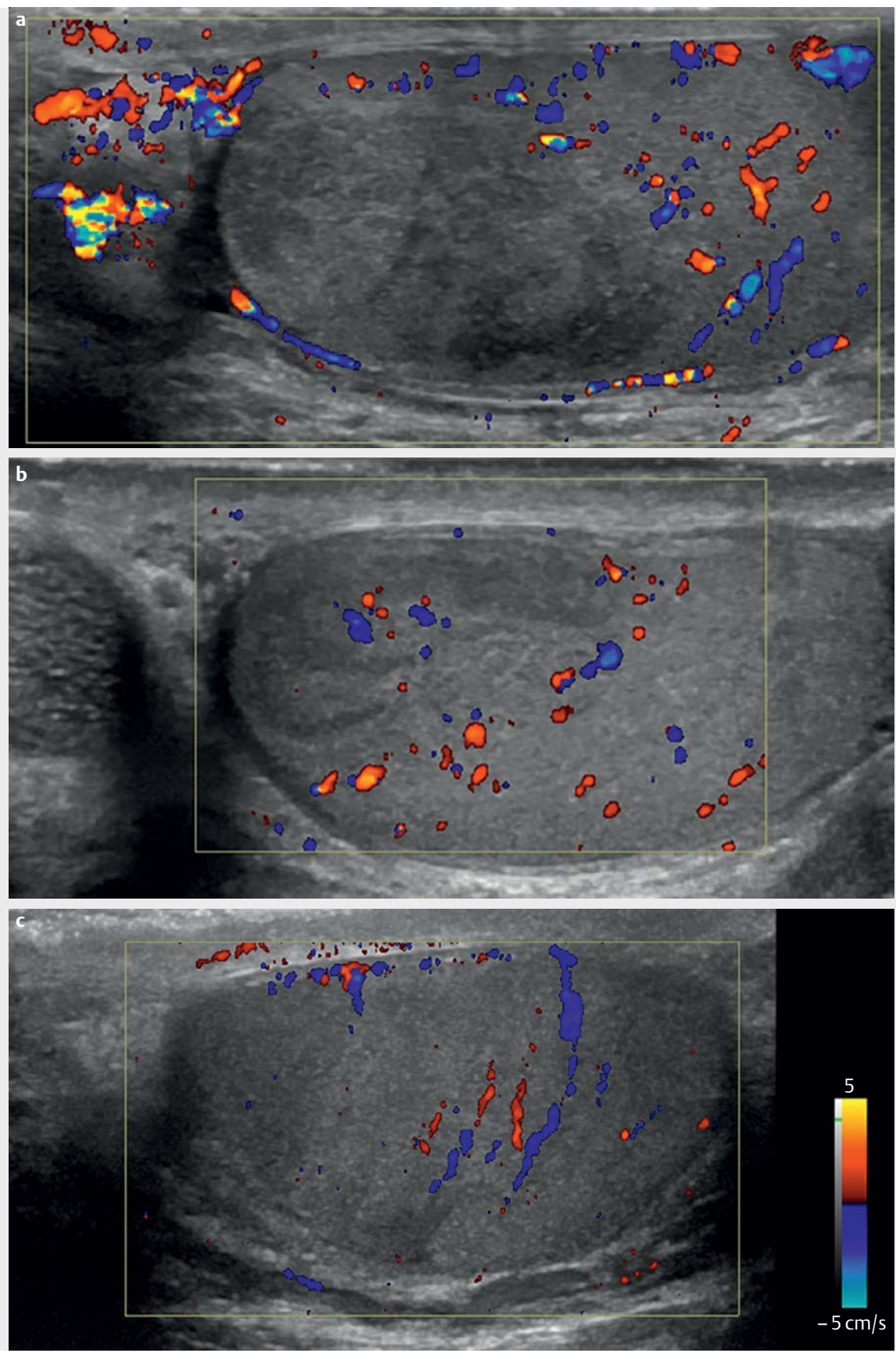

- Fig. 1 a Initial scan: B-mode and color Doppler ultrasonography of the left testicle, showing an inflamed left epididymis and a well-defined round inhomogeneous hypoechoic non-vascular lesion. b First follow-up ultrasonography after one month: Revealed normalization of the previous changes in the epididymis and the testicular lesion had reduced by more then $50 \%$ in size and was still hypoechoic and non-vascular. c Second follow-up ultrasonography after four months: Revealed focal atrophy in the upper pole of the testicle and slight return of vascularity. 
The majority of patients with testicular lesions undergo either surgical exploration or complete or partial unilateral orchiectomy due to patient concern, inconclusiveness of the US diagnosis, or a lack of knowledge regarding the diagnosis (EJ Street et al. Int ] STD AIDS 2017; 28(8): 744-749).

In this case report we present two examples of segmental testicular infarction with an emphasis on B-mode and color Doppler US as a follow-up modality.

\section{Case description}

\section{Case 1}

A 56-year-old male reported to the ER with severe pain in the left side of the scrotum for a few days. The patient reported no dysuria or history of scrotal trauma. Objective findings were mild reddening of the scro- tum and swelling of the left testicle and epididymis.

Infection parameters were slightly elevated and tumor markers (alpha-fetoprotein \& human chorionic gonadotropin (hCG)) were found to be normal. Bedside scrotal US performed by the urologist showed signs of epididymitis, and the patient was referred to the radiology department for confirmation of the diagnosis.
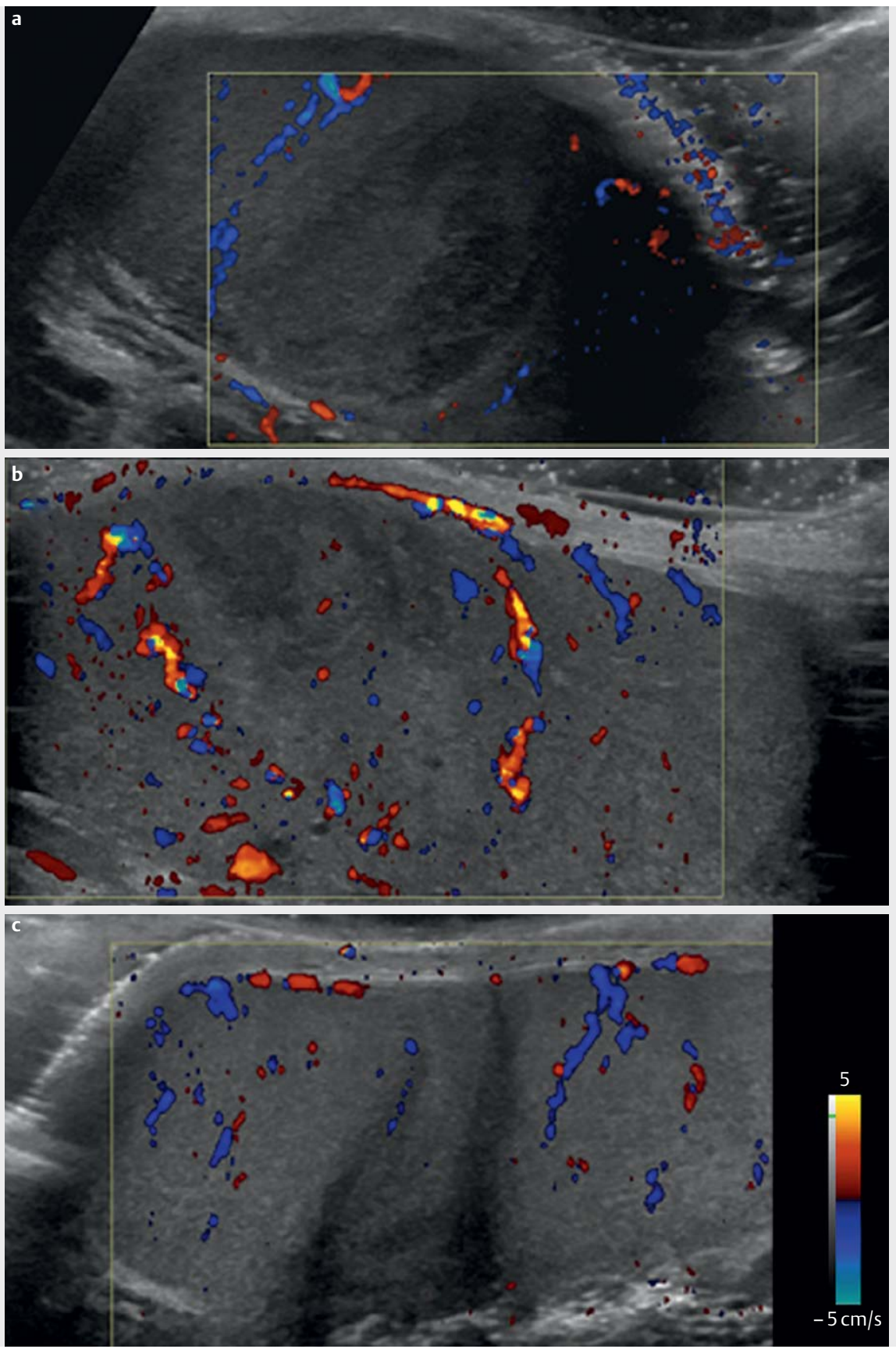

- Fig. 2 a Initial scan: B-mode and color Doppler ultrasonography of the left testicle, showing a well-defined inhomogeneous hypoechoic non-vascular lesion. b First follow-up ultrasonography after one month: Revealed normalization of the previous changes in the the testicular lesion which had reduced by more then $50 \%$ in size and was still hypoechoic and nonvascular. c Second follow-up ultrasonography after four months: Revealed focal atrophy in the middle of the left testis. 
B-mode and color Doppler US revealed an inflamed left epididymis and a well-defined, round inhomogeneous hypoechoic and non-vascular lesion in the upper pole of the left testicle. Segmental testicular infarction was suspected and a conservative approach with repeated biochemistry and follow-up scrotal US at one and four months was decided upon.

The first follow-up examination after one month revealed normalization of the previous changes in the epididymis and the testicular lesion had reduced by more than $50 \%$ in size and was still hypoechoic and non-vascular. The second follow-up examination at four months showed focal atrophy in the upper pole of the testicle and a slight return of vascularity ( $\triangleright$ Fig. 1). Furthermore, biochemistry findings normalized and tumor markers did not increase, thus segmental testicular infarction was the final diagnosis.

Case 2

A 25-year-old male under treatment for chlamydia came to the ER with acute severe pain in the left side of the scrotum. Objective findings were swelling and pronounced palpatory discomfort of the left testicle.

Infection parameters were slightly elevated and alpha-fetoprotein and hCG were normal. The patient was referred to the radiology department for an acute US examination as the diagnosis was inconclusive.

B-mode and color Doppler US revealed a well-defined wedge-shaped hypoechoic inhomogeneous and non-vascular lesion in the middle of the left testicle, involving approximately one third of the entire testicle. Segmental testicular infarction was suspected and a conservative approach with repeated biochemistry and scrotal US follow-up was decided upon.

Biochemistry findings normalized and tumor markers did not increase during follow-up. The first US after one month showed a decreasing size of the wedgeshaped hypoechoic non-vascular lesion. The four-month follow-up US examination showed focal atrophy of the left testicle ( Fig. 2). The final diagnosis was segmental testicular infarction.

\section{Discussion}

Segmental testicular infarction is a rare condition and only sporadically described in the medical literature. However, it is an important condition to consider since knowledge there- of may spare patients unnecessary surgery. Existing literature reveals that even though segmental testicular infarction is diagnosed based on B-mode and color Doppler US, most patients still undergo complete or partial unilateral orchiectomy (EJ Street et al. Int J STD AIDS 2017; 28(8): 744-749).

The initial US findings in the presented cases support previously described findings in related case reports. At the time of the two cases, we did not use contrast-enhanced ultrasound (CEUS) for testicular lesions at our institution. CEUS would definitely have helped us to diagnose the testicular infarction and avoid follow-up imaging. In a case report concerning a 23-year-old male, US showed a well-demarcated hypoechoic, non-vascular mass, but since the underlying cause was unclear the patient underwent partial orchiectomy. The subsequent pathologic examination revealed a diffuse hemorrhagic infarction (M Aquino et al. J Ultrasound Med 2013; 32(2): 365-372).

If the diagnosis of a testicular lesion is uncertain, other imaging modalities such as CEUS or MRI should be considered before surgical exploration is performed. The use of multi-parametric ultrasound, including CEUS and elastography, has also been described as useful in the diagnosis of segmental testicular infarction. The infarction is seen as an ischemic lobule, and a perilesional enhanced rim may be seen (V Cantisani Eur J Radiol 2015; 84(9): 1675 - 1684 and KV Patel et al. J Ultrasound 2014; 17(3): 233 -238). CEUS is used in daily practice worldwide and is an easy-to-use and indispensable diagnostic tool for non-hepatic applications (PS. Sidhu et al. Ultraschall Med 2018; 39(2): e2 - e44). MRI has been demonstrated to be useful for confirmation of the diagnosis. Testicular infarction is seen as a wedge-shaped infarction with the vertex towards the testicular mediastinum. T2 images show a well-defined border and contrast-enhanced T1 images show an enhanced rim surrounding the infarction (AC Tsili et al. Abdom Radiol 2019; 44(3): 1070-1082). MRI is expensive and time-consuming compared to multi-parametric ultrasound, including CEUS and elastography. Therefore, CEUS should be the first choice over MRI.

The most important differential diagnosis to consider regarding testicular infarction is malignancy. In general, a testicular tumor presents as a painless and palpable mass that appears vascular or hypervascular on US. Tumor markers are often elevated but do ap- pear normal in testicular seminoma (E] Street et al. Int J STD AIDS 2017; 28(8): 744-749).

In conclusion, segmental testicular infarction is a relatively uncommon and benign finding. Ultrasound including CEUS is an indispensable diagnostic tool to rule out testicular malignancy and avoid surgery, MRI, and follow-up imaging.

\section{Conflict of Interest}

The authors declare that they have no conflict of interest.

Authors

Camilla Brinkmann Bak-Ipsen ${ }^{1}$, Stine Degn $^{3}$, Linea Sandfeld Blichert-Refsgaard ${ }^{2}$, Tommy Kjærgaard Nielsen ${ }^{2}$, Henriette List $^{3}$, Ole Graumann ${ }^{3}$

\section{Affiliations}

1 Department of Radiology, Aarhus University Hospital, Aarhus

2 Department of Urology, Aarhus University Hospital, Aarhus

3 Department of Radiology, Odense University Hospital, Odense

\section{Correspondence}

Dr. Ole Graumann
Department of Radiology, Odense
Universitetshospital
Kløvervænget 10
5000 Odense
Denmark
Tel.: +4522910914
olegraumann@dadlnet.dk

Bibliography

Ultrasound Int Open 2020; 6: E50-E52

DOI 10.1055/a-1270-7795

ISSN 2199-7152

C 2020. The Author(s).

This is an open access article published by Thieme under the terms of the Creative Commons Attribution-NonDerivative-NonCommercial-License, permitting copying and reproduction so long as the original work is given appropriate credit. Contents may not be used for commecial purposes, or adapted, remixed, transformed or built upon. (https:// creativecommons.org/licenses/by-nc-nd/4.0/) Georg Thieme Verlag KG, Rüdigerstraße 14, 70469 Stuttgart, Germany

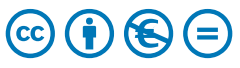

